\title{
Existence and controllability of fractional evolution equation with sectorial operator and impulse
}

\section{Di Zhang ${ }^{1 *}$ (D) and Yue Liang}

\section{"Correspondence:}

aqsy0008@163.com

'Science College of Gansu

Agricultural University, Lanzhou,

P.R. China

\begin{abstract}
This paper deals with the existence and uniqueness of PC-mild solutions for fractional impulsive evolution equation involving nonlocal conditions and sectorial operators. We also study the nonlocal controllability of the control system governed by fractional impulsive evolution equation.
\end{abstract}

MSC: 34A12; 34A37; 34H05

Keywords: Fractional evolution equation; Sectorial operators; Impulsive effects; Nonlocal conditions

\section{Introduction}

Let $X$ be a Banach space. Consider the nonlocal problem of the nonlinear fractional impulsive evolution equation of the form

$$
\left\{\begin{array}{l}
{ }^{C} D_{t}^{q} x(t)=\mathcal{A} x(t)+f(t, x(t)), \quad t \in J, t \neq t_{k}, \\
\left.\Delta x\right|_{t=t_{k}}=I_{k}\left(x\left(t_{k}\right)\right), \quad k=1,2, \ldots, m, \\
x(0)+g(x)=x_{0} \in X
\end{array}\right.
$$

where $J=[0, a], a>0$ is a constant, ${ }^{C} D_{t}^{q}$ denotes the Caputo fractional derivative of order $q \in(0,1), \mathcal{A}: D(\mathcal{A}) \subset X \rightarrow X$ is a sectorial operator in $X, t_{k}(k=1,2, \ldots, m)$ are the constants where the impulses occur, $0=t_{0}<t_{1}<t_{2}<\cdots<t_{m}<t_{m+1}=a,\left.\Delta x\right|_{t=t_{k}}=x\left(t_{k}^{+}\right)-x\left(t_{k}^{-}\right)$, $x\left(t_{k}^{+}\right)$and $x\left(t_{k}^{-}\right)$denote the right and left limits of $x$ at $t=t_{k}$, and $f, g$, and $I_{k}$ are given functions, which will be specified later.

It is well known that the fractional derivatives are valuable tools for description of memory and hereditary properties of various materials and processes, which cannot be characterized by integer-order derivatives. The fractional differential equations have gained considerable importance during the past three decades. Hence, the theory of fractional differential equations has emerged as an active branch of applied mathematics. It has been used to construct many mathematical models in various fields, such as physics, chemistry, electrodynamics of a complex medium, polymer rheology, and so on. For recent works on the theory and applications of fractional differential equations, we refer to the monographs $[12,20,26,28,29,32]$ and the papers $[1-7,10,11,13-19,21-25,27,30,31,33-42]$.

(c) The Author(s) 2018. This article is distributed under the terms of the Creative Commons Attribution 4.0 International License (http://creativecommons.org/licenses/by/4.0/), which permits unrestricted use, distribution, and reproduction in any medium, provided you give appropriate credit to the original author(s) and the source, provide a link to the Creative Commons license, and indicate if changes were made. 
In addition, impulsive differential equations have been an interesting object because of their wide applications in physics, biology, engineering, medical fields, industry, and technology. Impulsive differential problems are appropriate models for describing the real processes that change their states rapidly at certain moments and cannot be described by using the classical differential equations. The fractional differential equations with impulsive effects have been studied by many authors; see $[2,3,7,10,13-15,19,21,25,27,34$, $35,37]$ and the references therein.

Moreover, the nonlocal Cauchy problems, which have strong background coming from physical problems, were initialed by Byszewski $[8,9]$. Because nonlocal initial conditions generalize classical ones and play an important role in physics and engineering, more and more researchers pay attention to nonlocal Cauchy problems for different kinds of differential equations. For the fractional differential equations with nonlocal conditions, we refer to $[18,23,42]$. For the fractional differential equations with nonlocal conditions and impulsive effects, we refer to [13, 14, 21, 37].

Recently, the existence of mild solutions for abstract evolution equations or inclusions involving sectorial operators has been studied by many authors. For example, Shu et al. [34] introduced the concept of mild solutions for the initial value problem of fractional impulsive evolution equations (1.1). Assuming that $\mathcal{A}$ is a sectorial operator and $f$ is Lipschitz continuous or completely continuous, they proved the existence theorems of mild solutions for (1.1) when the operator families $\left(\mathcal{U}_{q}(t)\right)_{t \geq 0}$ and ${\overline{\left(\mathcal{V}_{q}(t)\right)_{t \geq 0}}}_{\text {, where }}$ ${\overline{\left(\mathcal{V}_{q}(t)\right)_{t \geq 0}}}=t^{1-q} \mathcal{V}_{q}(t)$, are compact. Agarwal et al. [1], in finite-dimensional spaces, discussed the existence of mild solutions for fractional nonlocal evolution inclusions without impulses when $\mathcal{A}$ is a sectorial operator. They studied the dimension of the set of mild solutions. Wang et al. [37] investigated the existence of PC-mild solutions for fractional impulsive evolution inclusions with nonlocal initial conditions when $\mathcal{A}$ is a sectorial operator. The results are obtained without supposing the compactness of operator families $\left(\mathcal{U}_{q}(t)\right)_{t \geq 0}$ and ${\overline{\left(\mathcal{V}_{q}(t)\right)}}_{t \geq 0}$, but the nonlinear multivalued function satisfies the regularity condition expressed by the measure of noncompactness.

In this paper, motivated by the results mentioned, we consider the existence and controllability of fractional nonlocal impulsive problem for abstract evolution equation (1.1). Our main result, Theorem 3.1, extends Theorem 3.1 of [34] by discussing the problem in a new set $\mathcal{S}$. Particularly, because of this set $\mathcal{S}$, Theorem 3.1 is not a particular case of Theorem 3.3 in [37]. At last, we study the nonlocal controllability of fractional impulsive evolution equation with nonlocal condition of the form (1.1) (see Theorem 3.4 for details), which is not discussed in [34, 37].

The rest of the paper is organized as follows. In Sect. 2, we recall some definitions and notions of sectorial operators. In Sect. 3, we prove the existence, uniqueness, and nonlocal controllability of PC-mild solutions for the fractional impulsive evolution equation (1.1).

\section{Preliminaries}

Let $X$ be a real Banach space with norm $\|\cdot\|$. We denote by $C(J, X)$ the Banach space of all continuous functions $x: J \rightarrow X$ with the norm $\|x\|_{C}=\sup \{\|x(t)\|: t \in J\}$. For any $p \in[1, \infty], L^{p}(J, X)$ denotes the Banach space of all strongly measurable functions $x: J \rightarrow X$ with the norm

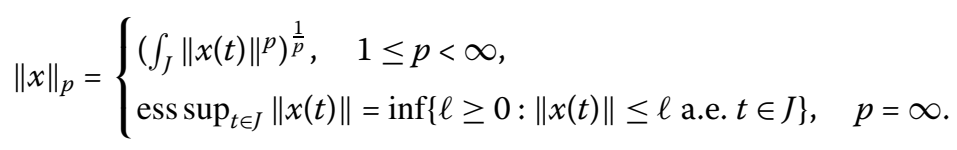


Let $L(X)$ be the Banach space of bounded linear operators from $X$ to $X$. For a linear operator $\mathcal{A}$, we denote by $\sigma(\mathcal{A})$ its spectrum and by $\rho(\mathcal{A})=\mathbb{C}-\sigma(\mathcal{A})$ its resolvent set. The family $R(\lambda, \mathcal{A})=(\lambda I-\mathcal{A})^{-1}, \lambda \in \rho(\mathcal{A})$, denotes the resolvent operator of $\mathcal{A}$.

An operator $\mathcal{A}$ is called a sectorial operator if

(1) $\rho(\mathcal{A}) \subset \sum_{\eta, \varrho}$, and

(2) $\|R(\lambda, \mathcal{A})\|_{L(X)} \leq \frac{M}{|\lambda-\rho|}, M>0, \lambda \in \sum_{\eta, \varrho}$,

where $\sum_{\eta, \varrho}=\{\lambda \in \mathbb{C}: \lambda \neq \varrho,|\arg (\lambda-\varrho)|<\eta\}, \eta \in\left[\frac{\pi}{2}, \pi\right]$, and $\varrho \in \mathbb{R}$. Examples of sectorial operators are some differential operators on unbounded domains, such as the Laplace operator or the Stokes operator on exterior domains.

Let $q>0$ and $\omega=[q]$, the smallest integer greater than or equal to $q$. Given $\widehat{x}_{0}$, we consider the Cauchy problem of the $q \in(\omega-1, \omega)$ th-order Caputo fractional evolution equation of the form

$$
\left\{\begin{array}{l}
{ }^{C} D_{t}^{q} x(t)=\mathcal{A} x(t), \quad t>0, \\
x(0)=\widehat{x}_{0}, \quad x^{(i)}(0)=0, \quad i=1,2, \ldots, \omega-1,
\end{array}\right.
$$

where $\mathcal{A}$ is a sectorial operator that is closed and densely defined in $X$. Definitions 2.1-2.3 and Lemma 2.4 can be found in $[4,26,28]$.

Definition 2.1 A family $\left\{\mathcal{U}_{q}(t)\right\}_{t \geq 0}$ is called a solution operator of the Cauchy problem (2.1) if the following conditions are satisfied:

(i) $\mathcal{U}_{q}(t)$ is strongly continuous for $t \geq 0$, and $\mathcal{U}_{q}(0)=I$, where $I$ is the identity operator;

(ii) $\mathcal{U}_{q}(t) D(\mathcal{A}) \subset D(\mathcal{A})$ and $\mathcal{A} \mathcal{U}_{q}(t) x=\mathcal{U}_{q}(t) \mathcal{A} x$ for all $x \in D(\mathcal{A})$ and $t \geq 0$;

(iii) $\mathcal{U}_{q}(t) x$ is a solution of (2.1) for all $x \in D(\mathcal{A})$ and $t \geq 0$.

Definition 2.2 The solution operator $\mathcal{U}_{q}(t)$ is called exponentially bounded if

$$
\left\|\mathcal{U}_{q}(t)\right\|_{L(X)} \leq C e^{v t}, \quad t \geq 0
$$

for some constants $C \geq 1$ and $v \geq 0$. An operator $\mathcal{A}$ is said to belong to $\mathfrak{e}^{q}(C, v)$ if the solution operator $\mathcal{U}_{q}(t)$ of (2.1) satisfies (2.2).

Denote $\mathfrak{e}^{q}(v):=\bigcup\left\{\mathfrak{e}^{q}(C, v): C \geq 1\right\}$ and $\mathfrak{e}^{q}:=\bigcup\left\{\mathfrak{e}^{q}(v): v \geq 0\right\}$. Clearly, $\mathfrak{e}^{1}$ and $\mathfrak{e}^{2}$ are the sets of all infinitesimal generators of $C_{0}$-semigroups and cosine operator families, respectively.

Definition 2.3 A solution operator $\mathcal{U}_{q}(t)$ of (2.1) is said to be analytic if it admits an analytic extension to a sector $\sum_{\eta_{0}}:=\left\{\lambda \in \mathbb{C}-\{0\}:|\arg \lambda|<\eta_{0}\right\}$ for some $\eta_{0} \in\left(0, \frac{\pi}{2}\right]$. An analytic solution operator of (2.1) is said to be of analyticity type $\left(\eta_{0}, \varrho_{0}\right)$ if for all $\eta<\eta_{0}$ and $\varrho>\varrho_{0}$, there is $C=C(\eta, \varrho)$ such that

$$
\left\|\mathcal{U}_{q}(t)\right\|_{L(X)} \leq C e^{v R e t}
$$

for some $t \in \sum_{\eta}:=\{t \in \mathbb{C}-\{0\}:|\arg t|<\eta\}$. 
Let us introduce $\mathcal{A}^{q}\left(\eta_{0}, \varrho_{0}\right):=\left\{\mathcal{A} \in \mathfrak{e}^{q}: \mathcal{A}\right.$ generates an analytic solution operator $\mathcal{U}_{q}(t)$ of analyticity type $\left.\left(\eta_{0}, \varrho_{0}\right)\right\}$. Furthermore, set

$$
\mathcal{A}^{q}\left(\eta_{0}\right):=\left\{\mathcal{A}^{q}\left(\eta_{0}, \varrho_{0}\right): \varrho_{0} \in \mathbb{R}\right\}, \quad \mathcal{A}^{q}:=\left\{\mathcal{A}^{q}\left(\eta_{0}\right): \eta_{0} \in\left(0, \frac{\pi}{2}\right]\right\} .
$$

For $q=1$, we obtain the set of all infinitesimal generators of analytic semigroups.

Lemma 2.4 Let $q \in(0,2)$. A linear closed densely defined operator $\mathcal{A}$ belongs to $\mathcal{A}^{q}\left(\eta_{0}, \varrho_{0}\right)$ iff $\lambda^{q} \in \rho(\mathcal{A})$ for each $\lambda \in \sum_{\eta_{0}+\frac{\pi}{2}}\left(\varrho_{0}\right):=\left\{\lambda \in \mathbb{C}-\{0\}:\left|\arg \left(\lambda-\varrho_{0}\right)\right|<\eta_{0}+\frac{\pi}{2}\right\}$ and $\varrho>\varrho_{0}$, $\eta<\eta_{0}$, there exists a constant $M=M(\eta, \varrho)$ such that

$$
\left\|\lambda^{q-1} R\left(\lambda^{q}, \mathcal{A}\right)\right\|_{L(X)} \leq \frac{M}{|\lambda-\varrho|}
$$

for some $\lambda \in \sum_{\eta_{0}+\frac{\pi}{2}}(\varrho)$.

If $\mathcal{A} \in \mathcal{A}^{q}\left(\eta_{0}, \varrho_{0}\right)$ for some $\eta_{0} \in\left(0, \frac{\pi}{2}\right]$ and $\varrho_{0} \in \mathbb{R}$, we see, from the proof of Theorem 2.14 in [4], that the solution operator of the Cauchy problem (2.1) is defined by

$$
\mathcal{U}_{q}(t)=\frac{1}{2 \pi i} \int_{\Gamma} e^{\tau t} \tau^{q-1} R\left(\tau^{q}, \mathcal{A}\right) d \tau,
$$

where $\Gamma$ is a suitable path lying on $\sum_{\eta, \varrho^{\circ}}$.

Let $J_{0}=\left[0, t_{1}\right], J_{i}=\left(t_{i}, t_{i+1}\right], i=1,2, \ldots, m$. We consider the space

$$
\begin{aligned}
P C(J, X)= & \left\{x: J \rightarrow X:\left.x\right|_{J_{i}} \in C\left(J_{i}, X\right), i=0,1,2, \ldots, m,\right. \\
& \text { and } \left.x\left(t_{i}^{+}\right) \text {exist for all } i=1,2, \ldots, m\right\} .
\end{aligned}
$$

Then $P C(J, X)$ is a Banach space with the norm

$$
\|x\|_{P C}=\sup \{\|x(t)\|: t \in J\}
$$

for $x \in P C(J, X)$. According to [1, 6, 34, 37], we have the following definition of PC-mild solutions for the nonlocal problem (1.1).

Definition 2.5 Let $\mathcal{A} \in \mathcal{A}^{q}\left(\eta_{0}, \varrho_{0}\right)$ for some $\eta_{0} \in\left(0, \frac{\pi}{2}\right]$ and $\varrho_{0} \in \mathbb{R}$. A function $x \in P C(J, X)$ is called a PC-mild solution of (1.1) if it satisfies the following integral equation:

$$
x(t)=\left\{\begin{array}{l}
\mathcal{U}_{q}(t)\left[x_{0}-g(x)\right]+\int_{0}^{t} \mathcal{V}_{q}(t-s) f(s, x(s)) d s, \quad t \in J_{0}, \\
\vdots \\
\quad \mathcal{U}_{q}(t)\left[x_{0}-g(x)\right]+\sum_{k=1}^{i} \mathcal{U}_{q}\left(t-t_{k}\right) I_{k}\left(x\left(t_{k}\right)\right) \\
\quad+\int_{0}^{t} \mathcal{V}_{q}(t-s) f(s, x(s)) d s, \quad t \in J_{i}, 1 \leq i \leq m,
\end{array}\right.
$$

where $\mathcal{U}_{q}(t)$ is given in (2.3), and

$$
\mathcal{V}_{q}(t)=\frac{1}{2 \pi i} \int_{\Gamma} e^{\tau t} R\left(\tau^{q}, \mathcal{A}\right) d \tau
$$

where $\Gamma$ is a suitable path lying on $\sum_{\eta, \varrho}$. 
Remark 1 Our Definition 2.5 follows from Definition 2.20 of [37]. In [37], the authors claimed that the definition of solutions of impulsive Caputo fractional equations is questionable. They do not claim that Definition 2.20 is the best one. I agree with their view. Therefore Definition 2.5 is following a similar way as for ordinary differential equations with impulses. For details of Definition 2.5, we refer to [15, 38].

By (2.3) and (2.4) the operator families $\left\{\mathcal{U}_{q}(t)\right\}_{t \geq 0}$ and $\left\{\mathcal{V}_{q}(t)\right\}_{t \geq 0}$ satisfy the following properties $[4,26,28]$.

Lemma 2.6 If $\mathcal{A} \in \mathcal{A}^{q}\left(\eta_{0}, \varrho_{0}\right)$ for some $\eta_{0} \in\left(0, \frac{\pi}{2}\right]$ and $\varrho_{0} \in \mathbb{R}$, then

$$
\left\|\mathcal{U}_{q}(t)\right\|_{L(X)} \leq \widehat{M}_{1}, \quad\left\|\mathcal{V}_{q}(t)\right\|_{L(X)} \leq \widehat{M}_{2} t^{q-1}
$$

where

$$
\widehat{M}_{1}=\sup _{t \in J}\left\|\mathcal{U}_{q}(t)\right\|_{L(X)}, \quad \widehat{M}_{2}=\sup _{t \in J} M e^{\varrho t}\left(1+t^{1-q}\right)
$$

where $M=M(\eta, \varrho)$ is a constant.

\section{Main results}

Let $\mathcal{S}:=\left\{x \in P C(J, X): \exists L>0,\|x(t)\| \leq L e^{\theta t}, \forall \theta>0\right.$, a.e. $\left.t \in J\right\}$. Define the norm

$$
\|x\|_{\mathcal{S}}=\sup \left\{\|x(t)\| e^{-\theta t}: t \in J\right\}
$$

It is easy to see that $\mathcal{S}$ is a Banach space with norm (3.1) and

$$
\|x\|_{\mathcal{S}} \leq L, \quad \forall x \in \mathcal{S}
$$

Theorem 3.1 Let $\mathcal{A} \in \mathcal{A}^{q}\left(\eta_{0}, \varrho_{0}\right)$ for some $\eta_{0} \in\left(0, \frac{\pi}{2}\right]$ and $\varrho_{0} \in \mathbb{R}$. Suppose that the following conditions hold:

(H1) $f: J \times X \rightarrow X$, and there exists a function $\rho \in L^{1}\left(J, \mathbb{R}^{+}\right)$such that

$$
\|f(t, u)-f(t, v)\| \leq \rho(t)\|u-v\|, \quad \text { a.e. } t \in J, u, v \in X
$$

(H2) For each $k \in\{1,2, \ldots, m\}, I_{k}: X \rightarrow X$, and there exists $\zeta_{k}>0$ such that

$$
\left\|I_{k}(u)-I_{k}(v)\right\| \leq \zeta_{k}\|u-v\|, \quad \forall u, v \in X
$$

(H3) $g: \mathcal{S} \rightarrow X$ is continuous, and there exists a constant $K>0$ such that

$$
\|g(x)-g(y)\| \leq K\|x-y\|_{\mathcal{S}}, \quad \forall x, y \in \mathcal{S} .
$$


(H4) There exist constants $\gamma_{1}>0$ and $\beta_{1} \in(0,1)$ such that

$$
\begin{aligned}
& \widehat{M}_{1}\left[\left\|x_{0}\right\|+\|g(0)\|+\sum_{k=1}^{m}\left\|I_{k}(0)\right\|\right]+\widehat{M}_{2} \int_{0}^{t}(t-s)^{q-1}\|f(s, 0)\| d s \\
& \quad \leq \gamma_{1} e^{\theta t}, \quad t \in J \\
& \widehat{M}_{1}\left[K+e^{a \theta} \sum_{k=1}^{m} \zeta_{k}\right]+\widehat{M}_{2} \int_{0}^{t}(t-s)^{q-1} \rho(s) e^{\theta s} d s \leq \beta_{1} e^{\theta t}, \quad t \in J .
\end{aligned}
$$

Then the nonlocal problem (1.1) has a unique PC-mild solution on J.

Proof Define the operator $Q: \mathcal{S} \rightarrow P C(J, X)$ by

$$
(Q x)(t)=\left\{\begin{array}{l}
\mathcal{U}_{q}(t)\left[x_{0}-g(x)\right]+\int_{0}^{t} \mathcal{V}_{q}(t-s) f(s, x(s)) d s, \quad t \in J_{0}, \\
\mathcal{U}_{q}(t)\left[x_{0}-g(x)\right]+\sum_{k=1}^{i} \mathcal{U}_{q}\left(t-t_{k}\right) I_{k}\left(x\left(t_{k}\right)\right) \\
\quad+\int_{0}^{t} \mathcal{V}_{q}(t-s) f(s, x(s)) d s, \quad t \in J_{i}, 1 \leq i \leq m
\end{array}\right.
$$

We first prove that $Q \mathcal{S} \in \mathcal{S}$. For any $x \in \mathcal{S}$ and $t \in J_{0}$, it follows from (H1)-(H4), (3.2), and Lemma 2.6 that

$$
\begin{aligned}
\|(Q x)(t)\| \leq & \left\|\mathcal{U}_{q}(t)\left[x_{0}-g(x)\right]\right\|+\left\|\int_{0}^{t} \mathcal{V}_{q}(t-s) f(s, x(s)) d s\right\| \\
\leq & \widehat{M}_{1}\left[\left\|x_{0}\right\|+\|g(x)\|\right]+\widehat{M}_{2} \int_{0}^{t}(t-s)^{q-1}\|f(s, x(s))\| d s \\
\leq & \widehat{M}_{1}\left\|x_{0}\right\|+\widehat{M}_{1} K\|x\|_{\mathcal{S}}+\widehat{M}_{1}\|g(0)\| \\
& +\widehat{M}_{2} \int_{0}^{t}(t-s)^{q-1} \rho(s)\|x(s)\| d s+\widehat{M}_{2} \int_{0}^{t}(t-s)^{q-1}\|f(x, 0)\| d s \\
\leq & \left(\gamma_{1}+L \beta_{1}\right) e^{\theta t} .
\end{aligned}
$$

Similarly, for any $t \in J_{i}, 1 \leq i \leq m$, we can prove that

$$
\|(Q x)(t)\| \leq\left(\gamma_{1}+L \beta_{1}\right) e^{\theta t}
$$

Thus, for all $t \in J$, we have

$$
\|(Q x)(t)\| \leq\left(\gamma_{1}+L \beta_{1}\right) e^{\theta t}
$$

This fact, combined with (3.1), implies

$$
\|Q x\|_{\mathcal{S}} \leq \gamma_{1}+L \beta_{1}
$$

Hence $Q x \in \mathcal{S}$ for all $x \in \mathcal{S}$. 
Next, we verify that $Q$ is a contraction. For any $t \in J_{0}$ and $x, y \in \mathcal{S}$, from (H1)-(H4), (3.2), and Lemma 2.6 we obtain

$$
\begin{aligned}
\|(Q x)(t)-(Q y)(t)\| & \leq\left\|\mathcal{U}_{q}(t)[g(x)-g(y)]\right\|+\left\|\int_{0}^{t} \mathcal{V}_{q}(t-s)[f(s, x(s))-f(s, y(s))] d s\right\| \\
& \leq \widehat{M}_{1}\|g(x)-g(y)\|+\widehat{M}_{2} \int_{0}^{t}(t-s)^{q-1}\|f(s, x(s))-f(s, y(s))\| d s \\
& \leq\left[\widehat{M}_{1} K+\widehat{M}_{2} \int_{0}^{t}(t-s)^{q-1} \rho(s) e^{\theta s} d s\right]\|x-y\|_{\mathcal{S}} \\
& \leq \beta_{1} e^{\theta t}\|x-y\|_{\mathcal{S}}
\end{aligned}
$$

Similarly, for any $x, y \in \mathcal{S}$ and $t \in J_{i}, 1 \leq i \leq m$, we have

$$
\begin{aligned}
& \|(Q x)(t)-(Q y)(t)\| \\
& \leq\left[\widehat{M}_{1} K+\widehat{M}_{1} e^{a \theta} \sum_{k=1}^{m} \zeta_{k}+\widehat{M}_{2} \int_{0}^{t}(t-s)^{q-1} \rho(s) e^{\theta s} d s\right]\|x-y\|_{\mathcal{S}} \\
& \leq \beta_{1} e^{\theta t}\|x-y\|_{\mathcal{S}} .
\end{aligned}
$$

Thus, for any $t \in J$ and $x, y \in \mathcal{S}$, we have

$$
\|(Q x)(t)-(Q y)(t)\| \leq \beta_{1} e^{\theta t}\|x-y\|_{\mathcal{S}} .
$$

This implies

$$
\|Q x-Q y\|_{\mathcal{S}} \leq \beta_{1}\|x-y\|_{\mathcal{S}}, \quad \forall x, y \in \mathcal{S} .
$$

Since $\beta_{1} \in(0,1)$, we obtain that $Q: \mathcal{S} \rightarrow \mathcal{S}$ is a contraction. Applying the Banach fixed point theorem, we get that the operator $Q$ has a unique fixed point in $\mathcal{S}$. Hence, the nonlocal problem (1.1) has a unique PC-mild solution in $\mathcal{S}$.

Next, we consider the controllability of control system governed by the fractional impulsive evolution equation

$$
\left\{\begin{array}{l}
{ }^{C} D_{t}^{q} x(t)=\mathcal{A} x(t)+f(t, x(t))+B u(t), \quad t \in J, t \neq t_{k}, \\
\left.\Delta x\right|_{t=t_{k}}=I_{k}\left(x\left(t_{k}\right)\right), \quad k=1,2, \ldots, m, \\
x(0)+g(x)=x_{0} \in X
\end{array}\right.
$$

where the notions ${ }^{C} D_{t}^{q}, \mathcal{A},\left.\Delta x\right|_{t=t_{k}}$ and the functions $f, I_{k}, g$ are defined as in (1.1), $u$ is given in $L^{\infty}(J, U)$, the Banach space of admissible control functions, $U$ is a real Banach space, and $B$ is a bounded linear operator from $U$ to $X$.

Definition 3.2 System (3.6) is said to be nonlocally controllable on the interval $J=[0, a]$ if for any $x_{0}, x_{1} \in X$, there exists a control function $u \in L^{\infty}(J, U)$ such that the PC-mild solution of (3.6) satisfies $x(0)=x_{0}-g(x)$ and $x(a)=x_{1}-g(x)$.

To prove the nonlocal controllability of system (3.6), we introduce the following assumptions: 
(H5) The bounded linear operator $W: L^{\infty}(J, X) \rightarrow X$ defined by

$$
W(u)=\int_{0}^{a} \mathcal{V}_{q}(a-s) B u(s) d s
$$

has a bounded inverse $W^{-1}: X \rightarrow L^{\infty}(J, X) / \operatorname{Ker}(W)$, and there exists a constant $N>0$ such that $\left\|W^{-1}\right\| \leq N$ and $\|B\| \leq N$.

(H6) There exist two positive constants $\gamma_{2}$ and $\beta_{2}$ satisfying

$$
\left(1+\widehat{M}_{2} N^{2} \frac{a^{q}}{q} e^{a \theta}\right) \beta_{2}<1
$$

such that

$$
\begin{aligned}
& N_{1}+\widehat{M}_{2} \int_{0}^{t}(t-s)^{q-1}\|f(s, 0)\| d s \leq \gamma_{2} e^{\theta t}, \quad t \in J, \\
& N_{2}+\widehat{M}_{2} \int_{0}^{t}(t-s)^{q-1} \rho(s) e^{\theta s} d s \leq \beta_{2} e^{\theta t}, \quad t \in J,
\end{aligned}
$$

where

$$
\begin{aligned}
& N_{1}=\left\|x_{1}\right\|+\widehat{M}_{1}\left(\left\|x_{0}\right\|+\sum_{k=1}^{m}\left\|I_{k}(0)\right\|\right)+\left(1+\widehat{M}_{1}\right)\|g(0)\|, \\
& N_{2}=\left(1+\widehat{M}_{1}\right) K+\widehat{M}_{1} e^{a \theta} \sum_{k=1}^{m} \zeta_{k} .
\end{aligned}
$$

In view of assumption (H5), we can define the control function $u(\cdot ; x) \in L^{\infty}(J, X)$ by

$$
\begin{aligned}
u(t ; x)= & W^{-1}\left[x_{1}-g(x)-\mathcal{U}_{q}(t)\left(x_{0}-g(x)\right)-\sum_{k=1}^{m} \mathcal{U}_{q}\left(a-t_{k}\right) I_{k}\left(x\left(t_{k}\right)\right)\right. \\
& \left.-\int_{0}^{a} \mathcal{V}_{q}(a-s) f(s, x(s)) d s\right](t), \quad t \in J .
\end{aligned}
$$

Lemma 3.3 If assumptions (H1)-(H3), (H5), and (H6) hold, then

$$
\|B u(t ; x)\| \leq N^{2}\left(\gamma_{2}+\beta_{2} L\right) e^{\theta a}
$$

and

$$
\|B u(t ; x)-B u(t, y)\| \leq N^{2} \beta_{2} e^{\theta a}\|x-y\|_{\mathcal{S}}
$$

for all $t \in J$ and $x, y \in \mathcal{S}$.

Proof For any $t \in J$ and $x \in \mathcal{S}$, it follows from assumptions (H1)-(H3), (H5), and (H6) that

$$
\begin{aligned}
\|B u(t ; x)\| \leq & N^{2}\left[\left\|x_{1}\right\|+\|g(x)\|+\widehat{M}_{1}\left(\left\|x_{0}\right\|+\|g(x)\|\right)+\widehat{M}_{1} \sum_{k=1}^{m}\left\|I_{k}\left(x\left(t_{k}\right)\right)\right\|\right. \\
& \left.+\widehat{M}_{2} \int_{0}^{a}(a-s)^{q-1}\|f(s, x(s))\| d s\right]
\end{aligned}
$$




$$
\begin{aligned}
\leq & N^{2}\left[\left\|x_{1}\right\|+\widehat{M}_{1}\left\|x_{0}\right\|+\left(1+\widehat{M}_{1}\right)\|g(0)\|\right. \\
& +\widehat{M}_{1} \sum_{k=1}^{m}\left\|I_{k}(0)\right\|+\widehat{M}_{2} \int_{0}^{a}(a-s)^{q-1}\|f(s, 0)\| d s \\
& +\left(1+\widehat{M}_{1}\right) K\|x\|_{\mathcal{S}}+\widehat{M}_{1} e^{\theta a} \sum_{k=1}^{m} \zeta_{k}\|x\|_{\mathcal{S}} \\
& \left.+\widehat{M}_{2} \int_{0}^{a}(a-s)^{q-1} \rho(s) e^{\theta s} d s\|x\|_{\mathcal{S}}\right] \\
\leq & N^{2}\left(\gamma_{2}+\beta_{2} L\right) e^{\theta a},
\end{aligned}
$$

and for all $t \in J$ and $x, y \in \mathcal{S}$, we have

$$
\begin{aligned}
\| B u(t ; x) & -B u(t, y) \| \\
\leq & N^{2}\left[\|g(x)-g(y)\|+\widehat{M}_{1}\|g(x)-g(y)\|+\widehat{M}_{1} \sum_{k=1}^{m}\left\|I_{k}\left(x\left(t_{k}\right)\right)-I_{k}\left(y\left(t_{k}\right)\right)\right\|\right. \\
& \left.+\widehat{M}_{2} \int_{0}^{a}(a-s)^{q-1}\|f(s, x(s))-f(s, y(s))\| d s\right] \\
\leq & N^{2}\left[K\left(1+\widehat{M}_{1}\right)\|x-y\|_{\mathcal{S}}+\widehat{M}_{1} \sum_{k=1}^{m} \zeta_{k} e^{\theta t}\|x-y\|_{\mathcal{S}}\right. \\
& \left.+\widehat{M}_{2} \int_{0}^{a}(a-s)^{q-1} \rho(s) e^{\theta s} d s\|x-y\|_{\mathcal{S}}\right] \\
\leq & N^{2} \beta_{2} e^{\theta a}\|x-y\|_{\mathcal{S}} .
\end{aligned}
$$

This completes the proof.

Theorem 3.4 Let $\mathcal{A} \in \mathcal{A}^{q}\left(\eta_{0}, \varrho_{0}\right)$ for some $\eta_{0} \in\left(0, \frac{\pi}{2}\right]$ and $\varrho_{0} \in \mathbb{R}$. Suppose that conditions (H1)-(H3), (H5), and (H6) hold. Then system (3.6) is nonlocally controllable on the interval J.

Proof Define the operator $\widetilde{Q}: \mathcal{S} \rightarrow P C(J, X)$ by

$$
(\widetilde{Q} x)(t)=\left\{\begin{array}{l}
\mathcal{U}_{q}(t)\left[x_{0}-g(x)\right]+\int_{0}^{t} \mathcal{V}_{q}(t-s)[f(s, x(s))+B u(s ; x)] d s, \quad t \in J_{0}, \\
\mathcal{U}_{q}(t)\left[x_{0}-g(x)\right]+\sum_{k=1}^{i} \mathcal{U}_{q}\left(t-t_{k}\right) I_{k}\left(x\left(t_{k}\right)\right) \\
\quad+\int_{0}^{t} \mathcal{V}_{q}(t-s)[f(s, x(s))+B u(s ; x)] d s, \quad t \in J_{i}, 1 \leq i \leq m .
\end{array}\right.
$$

It is clear that any fixed point of $\widetilde{Q}$ is a PC-mild solution of the nonlocal system (3.6) satisfying $x(0)=x_{0}-g(x)$ and $x(a)=x_{1}-g(x)$. Next, we prove that $\widetilde{Q}$ has a fixed point in $\mathcal{S}$. For this purpose, we first prove that $\widetilde{Q}: \mathcal{S} \rightarrow \mathcal{S}$ is continuous.

Indeed, in view of the continuity of all functions involved in (3.6), $\widetilde{Q}: \mathcal{S} \rightarrow P C(J, X)$ is continuous. On the other hand, by assumptions (H1)-(H3) and (H6) and by Lemma 3.3, 
a similar argument as in the proof of Theorem 3.1 shows that

$$
\|(\widetilde{Q} x)(t)\| \leq\left(\gamma_{2}+\beta_{2} L\right)\left(1+\widehat{M}_{2} N^{2} e^{\theta(a-t)} \frac{a^{q}}{q}\right) e^{\theta t}, \quad \forall t \in J, x \in \mathcal{S} .
$$

This implies

$$
\|\widetilde{Q} x\|_{\mathcal{S}} \leq\left(\gamma_{2}+\beta_{2} L\right)\left(1+\widehat{M}_{2} N^{2} e^{\theta a} \frac{a^{q}}{q}\right)
$$

Thus $\widetilde{Q}: \mathcal{S} \rightarrow \mathcal{S}$ is continuous.

Secondly, we claim that $\widetilde{Q}: \mathcal{S} \rightarrow \mathcal{S}$ is a contraction. In fact, for any $t \in J_{0}$ and $x, y \in \mathcal{S}$, it follows from (H1)-(H3), (H6), and Lemma 3.3 that

$$
\begin{aligned}
\|(\widetilde{Q} x)(t)-(\widetilde{Q} y)(t)\| \leq & \widehat{M}_{1}\|g(x)-g(y)\| \\
& +\widehat{M}_{2} \int_{0}^{t}(t-s)^{q-1}\|f(s, x(s))-f(s, y(s))\| \\
& +\widehat{M}_{2} \int_{0}^{t}(t-s)^{q-1}\|B u(s ; x)-B u(s ; y)\| \\
\leq & \left(\widehat{M}_{1} K+\widehat{M}_{2} \int_{0}^{t}(t-s)^{q-1} \rho(s) e^{\theta s} d s\right)\|x-y\|_{\mathcal{S}} \\
& +\widehat{M}_{2} N^{2} \beta_{2} e^{a \theta} \frac{a^{q}}{q}\|x-y\|_{\mathcal{S}} \\
\leq & \left(1+\widehat{M}_{2} N^{2} e^{\theta(a-t)} \frac{a^{q}}{q}\right) \beta_{2} e^{\theta t}\|x-y\|_{\mathcal{S} .}
\end{aligned}
$$

Similarly, for any $t \in J_{i}, 1 \leq i \leq m$, and $x, y \in \mathcal{S}$, we can obtain

$$
\|(\widetilde{Q} x)(t)-(\widetilde{Q} y)(t)\| \leq\left(1+\widehat{M}_{2} N^{2} e^{\theta(a-t)} \frac{a^{q}}{q}\right) \beta_{2} e^{\theta t}\|x-y\|_{\mathcal{S}} .
$$

Thus, for all $x, y \in \mathcal{S}$, we have

$$
\begin{aligned}
\|\widetilde{Q} x-\widetilde{Q} y\|_{\mathcal{S}} & \leq\left(1+\widehat{M}_{2} N^{2} e^{\theta(a-t)} \frac{a^{q}}{q}\right) \beta_{2}\|x-y\|_{\mathcal{S}} \\
& \leq\left(1+\widehat{M}_{2} N^{2} e^{a \theta} \frac{a^{q}}{q}\right) \beta_{2}\|x-y\|_{\mathcal{S}} .
\end{aligned}
$$

By (3.7), $\widetilde{Q}: \mathcal{S} \rightarrow \mathcal{S}$ is a contraction. Hence, by the Banach fixed point theorem, $\widetilde{Q}$ has a unique fixed point $x$ in $\mathcal{S}$, which is a PC-mild solution of the nonlocal system (3.6) and satisfies $x(0)=x_{0}-g(x)$ and $x(a)=x_{1}-g(x)$.

\section{Conclusion and future study}

This paper deals with the existence and controllability of a fractional nonlocal impulsive problem for abstract evolution equation (1.1). By using fixed point theorems the existence and nonlocal controllability of (1.1) are discussed. A similar technique can be used to study the fractional evolution inclusion involving impulses and nonlocal condition. 


\section{Acknowledgements}

The authors are grateful to the referee for helpful comments and suggestions.

\section{Funding}

The work is supported by Shengtongsheng Foundation for Science and Technology Innovation (No. GSAU-STS-1540) and Gansu Technology Plan (No. 17JR5RA071).

\section{Competing interests}

All the authors have no any competing interests in the manuscript.

\section{Authors' contributions}

All authors contributed equally in writing this paper. All authors read and approved the final manuscript.

\section{Publisher's Note}

Springer Nature remains neutral with regard to jurisdictional claims in published maps and institutional affiliations.

Received: 21 January 2018 Accepted: 16 May 2018 Published online: 22 June 2018

\section{References}

1. Agarwal, R., Ahmad, B., Alsaedi, A., Shahzad, N.: Existence and dimension of the set of mild solutions to semilinear fractional differential inclusions. Adv. Differ. Equ. 2012, 74 (2012)

2. Agarwal, R., Benchohra, M., Slimani, B.: Existence results for differential equations with fractional order and impulses. Mem. Differ. Equ. Math. Phys. 44, 1-21 (2008)

3. Agarwal, R., Hristova, S., O'Regan, D.: A survey of Lyapunov functions, stability and impulsive Caputo fractional differential equations. Fract. Calc. Appl. Anal. 19, 290-318 (2016)

4. Agarwal, R., Lakshmikantham, V., Nieto, J.: On the concept of solutions for fractional differential equations with uncertainly. Nonlinear Anal. 72, 2859-2862 (2010)

5. Ahmed, H.: Approximate controllability of impulsive neutral stochastic differential equations with fractional Brownian motion in a Hilbert space. Adv. Differ. Equ. 2014, 113 (2014)

6. Bajlekova, E.: Fractional evolution equations in Banach spaces. Ph.D. thesis, Eindhoven University of Technology (2001)

7. Benchohra, M., Berhoun, F.: Impulsive fractional differential equations with variable times. Comput. Math. Appl. 59, $1245-1252(2010)$

8. Byszewski, L.: Theorems about existence and uniqueness of solutions of a semi-linear evolution nonlocal Cauchy problem. J. Math. Anal. Appl. 162, 494-505 (1991)

9. Byszewski, L., Lakshmikantham, V.: Theorems about the existence and uniqueness of solutions of a nonlocal Cauchy problem in a Banach space. Appl. Anal. 40, 11-19 (1990)

10. Cao, J., Chen, H.: Impulsive fractional differential equations with nonlinear conditions. Math. Comput. Model. 55 303-311 (2012)

11. Chatzarakis, G., Li, T.: Oscillation criteria for delay and advanced differential equations with non-monotone arguments. Complexity 2018, Article ID 8237634 (2018)

12. Das, S.: Functional Fractional Calculus. Springer, Berlin (2011)

13. Debbouche, A., Baleanu, D.: Controllability of fractional evolution nonlocal impulsive quasilinear delay integro-differential systems. Comput. Math. Appl. 62, 1442-1450 (2011)

14. Fan, Z.: Impulsive problems for semilinear differential equations with nonlocal conditions. Nonlinear Anal. 72, 1104-1109 (2010)

15. Fečkan, M., Zhou, Y., Wang, J.: On the concept and existence of solution for impulsive fractional differential equations Commun. Nonlinear Sci. Numer. Simul. 17, 3050-3060 (2012)

16. Feng, Q., Meng, F.: Explicit solutions for space-time fractional partial differential equations in mathematical physics by a new generalized fractional Jacobi elliptic equation-based subequation method. Optik 127, 7450-7458 (2016)

17. Feng, Q., Meng, F.: Traveling wave solutions for fractional partial differential equations arising in mathematical physics by an improved fractional Jacobi elliptic equation method. Math. Methods Appl. Sci. 40, 3676-3686 (2017)

18. Ghour, S., Omari, A.: On the existence of the mild solution for semilinear nonlocal fractional Cauchy problem. J. Nonlinear Sci. Appl. 9, 3348-3353 (2016)

19. Guo, T., Zhang, K.: Impulsive fractional partial equations. Appl. Math. Comput. 257, 581-590 (2015)

20. Hilfer, R.: Application of Fractional Calculus in Physics. World Scientific, Singapore (2000)

21. Ibrahim, A., Sarori, N.: Mild solutions for nonlocal impulsive fractional semilinear differential inclusions with delay in Banach spaces. Appl. Math. 4, 40-56 (2013)

22. Li, T., Rogovchenko, Y.: Oscillation criteria for second-order superlinear Emden-Fowler neutral differential equations. Monatshefte Math. 184, 489-500 (2017)

23. Liang, J., Yang, H.: Controllability of fractional integro-differential evolution equations with nonlocal conditions. Appl. Math. Comput. 254, 20-29 (2015)

24. Lukashchuk, S.: An approximate solution method for ordinary fractional differential equations with the Riemann-Liouville fractional derivatives. Commun. Nonlinear Sci. Numer. Simul. 19, 390-400 (2014)

25. Mahto, L., Abbas, S.: PC-almost automorphic solution of impulsive fractional differential equations. Mediterr. J. Math. 12, 771-790 (2015)

26. Miller, K., Ross, B.: An Introduction to the Fractional Calculus and Fractional Differential Equations. Wiley, New York (1993)

27. Mophou, G.: Existence and uniqueness of mild solutions to impulsive fractional differential equations. Nonlinear Anal. 72, 1604-1615 (2010)

28. Oldham, K., Spanier, J.: The Fractional Calculus. Academic Press, New York (1974) 
29. Podlubny, I.: Fractional Differential Equations. Mathematics in Science and Engineering, vol. 198. Academic Press, San Diego (1999)

30. Qin, H., Gu, Z., Fu, Y., Li, T.: Existence of mild solutions and controllability of fractional impulsive integrodifferential systems with nonlocal conditions. J. Funct. Spaces 2017, 1-11 (2017)

31. Qin, H., Zhang, C., Li, T., Chen, Y.: Controllability of abstract fractional differential evolution equations with nonlocal conditions. J. Math. Comput. Sci. 17, 293-300 (2017)

32. Sabatier, J., Agarwal, O., Machado, J.: Advances in Fractional Calculus: Theoretical Developments and Applications in Physics and Engineering. Springer, Berlin (2007)

33. Shao, J., Zheng, Z., Meng, F.: Oscillation criteria for fractional differential equations with mixed nonlinearities. Adv. Differ. Equ. 2013, 323 (2013)

34. Shu, X., Lai, Y., Chen, Y.: The existence of mild solutions for impulsive fractional partial differential equations. Nonlinear Anal. 74, 2003-2011 (2011)

35. Tai, Z., Wang, X.: Controllability of fractional-order impulsive neutral functional infinite delay integrodifferential systems in Banach spaces. Appl. Math. Lett. 22, 1760-1765 (2009)

36. Wang, J., Ahmed, H.: Null controllability of nonlocal Hilfer fractional stochastic differential equations. Miskolc Math. Notes 18, 1073-1083 (2017)

37. Wang, J., Ibrahim, A., Fečkan, M.: Nonlocal impulsive fractional differential inclusions with fractional sectorial operators on Banach spaces. Appl. Math. Comput. 257, 103-118 (2015)

38. Wang, J., Zhou, Y., Fečkan, M.: Abstract Cauchy problem for fractional differential equations. Nonlinear Dyn. 71, 685-700 (2013)

39. Wei, Z., Che, J.: Initial value problems for fractional differential equations involving Riemann-Liouville sequential fractional derivative. J. Math. Anal. Appl. 367, 260-272 (2010)

40. Xu, R.: Oscillation criteria for nonlinear fractional differential equations. J. Appl. Math. 2013, 1-7 (2013)

41. Yang, H., Ibrahim, E., Ma, J.: Hybrid fixed point theorems with application to fractional evolution equations. J. Fixed Point Theory Appl. 19, 2663-2679 (2017)

42. Zhou, Y., Jiao, F.: Nonlocal Cauchy problem for fractional evolution equations. Nonlinear Anal. 11, 4465-4475 (2010)

\section{Submit your manuscript to a SpringerOpen ${ }^{\circ}$ journal and benefit from:}

- Convenient online submission

- Rigorous peer review

- Open access: articles freely available online

- High visibility within the field

- Retaining the copyright to your article

Submit your next manuscript at $\gg$ springeropen.com 